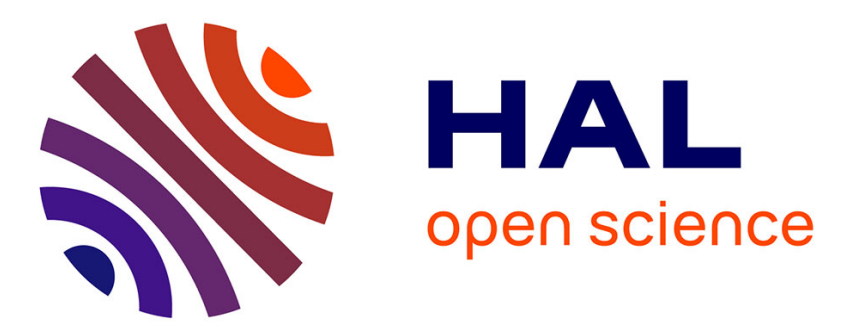

\title{
Nanometer optical trap based on stimulated emission in evanescence of a totally reflected Arago spot: Nanometer optical trap for fluorescent nanoparticles
}

\author{
O. Emile, Janine Emile
}

\section{- To cite this version:}

O. Emile, Janine Emile. Nanometer optical trap based on stimulated emission in evanescence of a totally reflected Arago spot: Nanometer optical trap for fluorescent nanoparticles. European Physical Journal E: Soft matter and biological physics, 2020, 43 (10), pp.68. 10.1140/epje/i2020-11991-6 . hal-03003159

\section{HAL Id: hal-03003159 \\ https://hal.science/hal-03003159}

Submitted on 20 Nov 2020

HAL is a multi-disciplinary open access archive for the deposit and dissemination of scientific research documents, whether they are published or not. The documents may come from teaching and research institutions in France or abroad, or from public or private research centers.
L'archive ouverte pluridisciplinaire HAL, est destinée au dépôt et à la diffusion de documents scientifiques de niveau recherche, publiés ou non, émanant des établissements d'enseignement et de recherche français ou étrangers, des laboratoires publics ou privés. 


\title{
Nanometer optical trap based on stimulated emission in evanescence of a totally reflected Arago spot.
}

\author{
Olivier EMILE \\ Université de Rennes 1, Campus de Beaulieu, F-35000 Rennes, France* \\ Janine EMILE \\ Université de Rennes 1, CNRS IPR UMR 6251, F-35000 Rennes, France
}

\begin{abstract}
Optical tweezers have paved the way towards the manipulation of particles and living cells at the micrometer range. Its extension towards the nanometer world may create unprecedented potentialities in many areas of science. Following a letter [O. Emile, J. Emile, and H. Tabuteau, EPL 129 (2020) 58001] that reported the observation of the trapping of a single 200-nm-diameter fluorescent particle in a nanometric volume, we detail here our experimental findings. In particular, the trapping mechanism is shown to be based on the radiation pressure of light in one direction and on the stimulated emission of the particle in the evanescent wave of a nanometer Arago spot on a glass/liquid interface on the other directions. The trapping volume is a 200-nm-height cylinder whose radius varies with the spreading of the evanescent wave near the spot and can reach $50 \mathrm{~nm}$. The calculation of the force and the parameters limiting the lifetime are detailed. Applications to laser trapping of atoms and molecules are also discussed.
\end{abstract}

\section{INTRODUCTION}

Since their first report in $1986[1,2]$, optical tweezers have become an exponentially growing technique used in optical manipulation of micrometer to nanometer particles [3-7]. These particles are trapped at the focal point of a tightly focused by optical gradient forces. Several applications of this non-contact particle manipulation have been considered ranging from movement tracking [8-10], application or detection of small forces [11, 12], altering cell membranes [13-15], cells sorting [16-18] and particles manipulation [19-24], to name a few. This last application, which is the most popular one, includes dielectric spheres, viruses, bacteria, organelles, or DNA stands manipulation. Optical tweezers and more generally optical manipulation, because of the tremendous development of nanotechnologies, now face a new challenge, that is extending its application range down to the sub-wavelength and nanometer scales world [25-29]. However, on the one hand, due to light diffraction, optical tweezer trap sizes are limited to a fraction of micrometer [30, 31], and, on the other hand, the trapping force diminishes as the third power of the particle diameter $[32,33]$, thus limiting their applications.

In order to circumvent these limitations, two main bypaths have been followed. The first one is based on a tight focused beam that is totally reflected on a glass/liquid interface [34-38], or on the evanescence of a guided optical beam [39-42]. However, whereas the trapping in the direction of the evanescent wave can reach nanometer sizes, the dimension of the light beam in the other directions is still limited by diffraction. The other direction explores the use of plasmon enhanced resonances from nanostructures [43-46], or at the end of optical fibres [47-50], or

* emile@univ-rennes1.fr the use of structured light fields [51-53], in order to break the diffraction limit imposed by focused laser beams.

Recently, we took advantage of the light interaction properties of fluorescent colloids to investigate the trapping of nanometer fluorescent particles in a so-called "Arago spot", totally reflected on a glass/liquid interface [54]. We have argued that the trapping mechanism relies on the stimulated emission of light in the evanescent wave of the totally reflected spot. The aim of this article is thus to give experimental evidence of the trapping mechanism and to detail it, based on new experimental observations.

The article is organized in as following. The first section (sect. II) is dedicated to the description of the experimental set up. The next section (sect. III) deals with the experimental results concerning the trap. The trapping mechanism together with its dynamics and its size are detailed in the following section (sect. IV). Finally, perspectives and possible applications are discussed in the last section (sect. V), before reaching the conclusion.

\section{EXPERIMENTAL SET UP}

\section{A. Description of the experiment}

The experimental set up has been described previously in [54], however for the self-consistency of the article let us recall it and complete it (see Fig. 1). A collimated laser beam (L4Cc Oxxius, wavelength $\lambda=488 \mathrm{~nm}$, power $250 \mathrm{~mW}$ at the end of an optical fibre) is diffracted by an occulting chromium disk (diameter $d=650,700$ or 750 $\mu \mathrm{m}$ ) deposited on a $e=170 \mu \mathrm{m}$ glass lamella (optical index $n_{g}=1.51$ ). The contour roughness of the disk is better than $100 \mathrm{~nm}$. The size of the beam is typically slightly higher than the size of the disk. The diffracted pattern is a so-called Arago spot [55] that is in total re- 
flection conditions on a glass/liquid interface (see Fig. 1b). This spot constitutes the trapping beam.

We determine the position of the spot at the glass/liquid interface using the following procedure (see Fig. 1c). We first image the spot using laser beam with a power diminished down to $2 \mathrm{~mW}$, at several distances below the interface (500 $\mu \mathrm{m}$ to $100 \mu \mathrm{m})$, where the spot size is in the tens micrometer range and is thus visible. The rays corresponding to this spot are not under total internal reflection conditions on the interface, part of the light is transmitted. Its position in the horizontal plane varies linearly with the distance from the interface. As we get closer to the interface, the spot size gets smaller. On the interface, the spot is associated with an angle of incidence equal to the critical angle. Then, by extrapolation, we define a $10 \mu \mathrm{m}$-diameter area on the interface where we expect the total reflection of the Arago spot to occur. We concentrate the investigation of particles in this area only.

The particles to be trapped are fluorescent polystyren spheres (Molecular Probes, diameter $2 a=200 \mathrm{~nm}$, optical index $n_{0}=1.51$ ) suspended in demineralized water (optical index $n_{l}=1.33$ ), flowing in a $30 \mu \mathrm{m}$-height, 300 $\mu \mathrm{m}$-width PDMS (PolyDiMethilSiloxane) micro channel made by standard soft lithography [56]. The channel is covered by a glass lamella with the mask deposited on it. The flow can be varied and controlled with a microfluidic pump. The particles are excited from underneath with an optical diode (ThorLabs M470L2, $470 \mathrm{~nm}$ central wavelength, power $700 \mathrm{~mW}$, focused to a $1 \mathrm{~mm}$ diameter spot on the channel). We image the trap with an inverted microscope (Leica, X20 objective lens, Numerical Aperture (NA) 0.4) equipped with a motorized XY stage. Pictures are taken every $30 \mathrm{~ms}$ by a SCMOS monochrome grayscale camera (Orca flash 4.0 Hamamatsu) and analyzed using ImageJ software [57].

\section{B. Experimental parameters}

The fluorescent particles consist of $N=1.1 \times 10^{5} \mathrm{flu}-$ orescein molecules (lifetime $\tau_{f}=4.1 \mathrm{~ns}$ ), leading to a fluorescent duty cycle (absorption followed by a spontaneous emission process) of $\Gamma=(N s) /\left[\tau_{f}(1+s)\right] \simeq 2 \times 10^{13}$ $\mathrm{s}^{-1}$ with a saturation of the transition of $s=2$.

The size of the trap depends on the size of the trapping beam. Actually, Arago spots can reach sizes smaller than usual Gaussian beams. The intensity distribution at the glass/liquid interface can be written as [58]

$$
I(r)=I_{0} \frac{e^{2}}{e^{2}+(d / 2)^{2}} J_{0}^{2}\left(\frac{\pi r d}{\lambda\left[e^{2}+(d / 2)^{2}\right]^{1 / 2}}\right)
$$

$J_{0}$ being the zeroth order Bessel function of the first kind, $r$ is the distance to the beam axis, $\lambda$ is the light wavelength, and $I_{0}$ is the intensity of the incoming light at the edges of the disk. The maximum light intensity of the spot at the glass/liquid interface is of the order of $I(r=0)=40 \mathrm{~mW} \cdot \mathrm{mm}^{-2}$. We characterize its size by its waist $w_{0}$, as for Gaussian beams. It is the radius at which the intensity values fall to $1 / e^{2}$ of its axial value [59]. Here, it equals to $w_{0}=120 \mathrm{~nm}$, which would be half of the waist of a strongly focused Gaussian beam of the same wavelength, focused by a lens of a numerical aperture equal to 1 . However, in the case of a Gaussian beam, there is a large spreading of the direction of the wavevector $k=2 \pi / \lambda$, whereas, in our case, at a given distance on the optical axis, the angle between the direction of propagation and the wavevectors is same. These wavevectors forming the Arago spot lie on the surface of a cone of angle $i$ (see Fig. 2).

This angle $i$ is the angle of incidence of the light beam on the glass/liquid interface. It equals $i=\tan ^{-1}(d / 2 e)=$ $62.39^{\circ}$ and is higher than (but close to) the critical angle $i_{c}=\sin ^{-1}\left(n_{w} / n_{g}\right)=61.74^{\circ}$. The Arago spot is thus totally reflected on the interface. Let us have a closer look at the rays impinging on the disk. Around the Arago spot, we have identified three types of rays: i) those impinging with an angle of incidence $i$ higher than (but very close to) the critical angle $i_{c}$ (Fig. 2a). ii) Those with $i>i_{c}$ (Fig. 2b). iii) Those with $i<i_{c}$ which are hardly transmitted but mostly reflected with no evanescence.

Let us detail the way those rays behave at the interface close to the Arago spot. In Fig. 2a, the light rays corresponding to the case i) lie down on the surface of a cone and lead to an Arago spot just on the glass/liquid interface. These rays are totally reflected on the interface. They generate an evanescent wave running away from the spot. The evanescent wave exists on a distance corresponding to the so-called Goos-Hänchen shift $\delta_{G-H}[60]$. It forms a "thick" donut which empty center part corresponds exactly to the Arago spot. In Fig. $2 \mathrm{~b}$, corresponding to the case ii), the rays form an Arago spot above the glass/liquid interface. The associated rays impinging on the surface are also under total reflection condition. The evanescent waves last on a smaller distance since $\delta_{G-H}$ is smaller (the angle of incidence is higher). They form a smaller donut with a bigger empty center. Besides, they interfere destructively on the interface. They play a little role on the trapping mechanism. The rays of case iii) are not under total internal reflexion condition when impinging on the interface. However, because of the laws of refraction close to the critical angle, the transmission coefficient is very low and only a very little part of the light is transmitted [55]. Most of the rays are reflected with no evanescence. Those rays hardly contribute to the electromagnetic field in the liquid medium, close to the interface. They also interfere destructively on the interface.

For a diffracting disk with a higher diameter, part of the angles of incidence below $i(\tan i=d / 2 e)$ are also under total internal reflection condition on the glass/liquid interface. They could form an ingoing evanescent wave that could be detrimental for the trapping. However, they interfere destructively on the interface and participate only marginally to the electric field. This is also true with the rays with an angle above $i$. For those with 


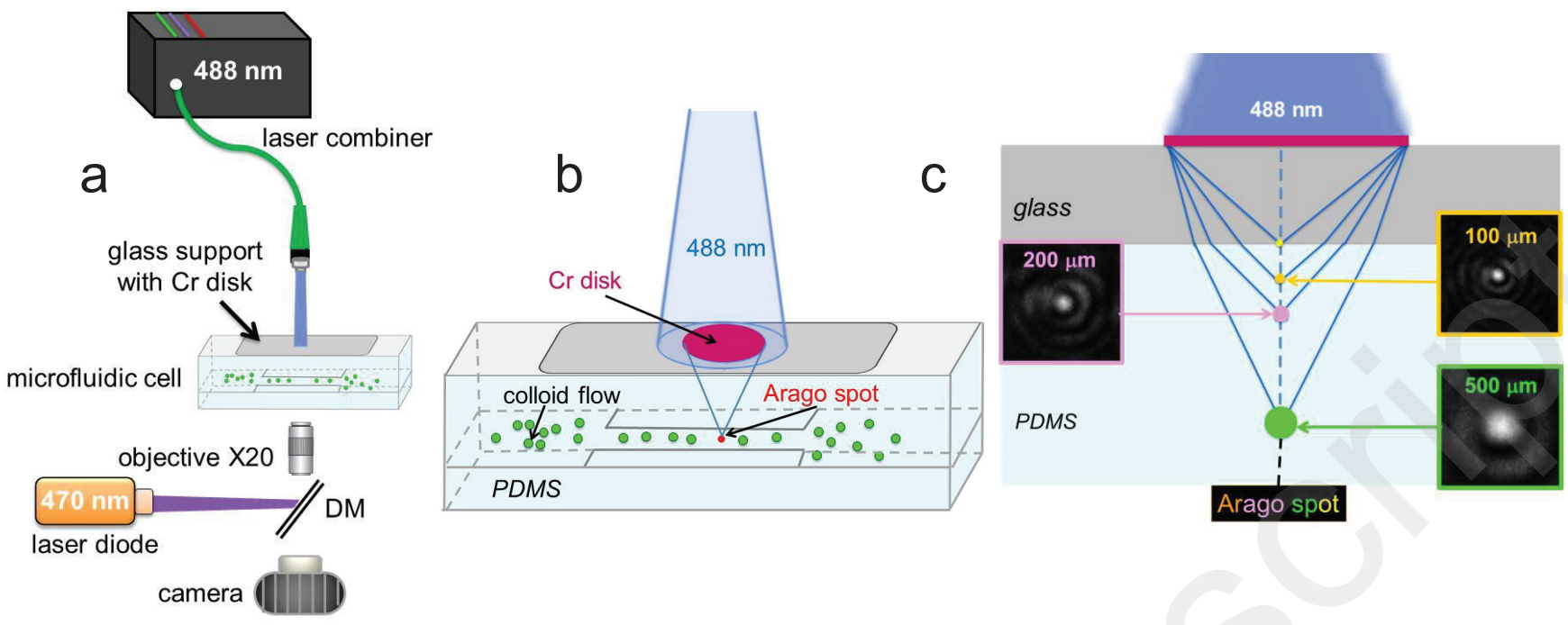

FIG. 1. Experimental set up: a) an Arago spot originates from the diffraction of a $488 \mathrm{~nm}$ collimated laser beam diffracted by a chromium disk deposited on a glass lamella. $200 \mathrm{~nm}$ diameter fluorescent colloids flowing within the liquid, are exited with a $470 \mathrm{~nm}$ diode. The fluorescence is collected with a X20 microscope objective and a camera. DM: Dichroic Mirror. b) Details of the experimental set-up. The Arago spot is under total internal reflection on the glass/liquid interface. The particles are trapped by the evanescent Arago spot. c) Propagation of the Arago spot in the PDMS underneath the channel. By extrapolation, this enables the localization of the spot on the interface.

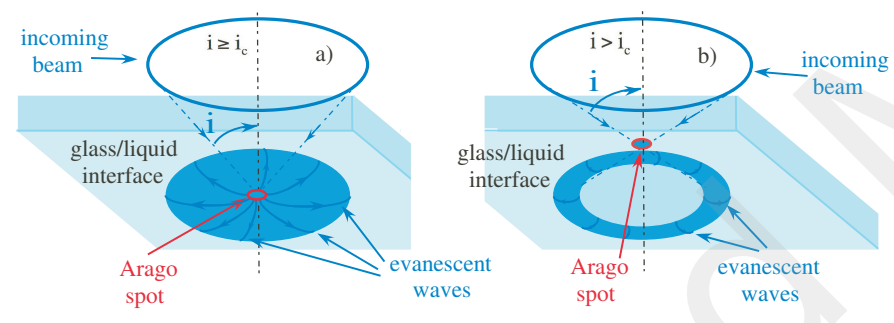

FIG. 2. a) For $\tan i=d / 2 e\left(i\right.$ close to the critical angle $\left.i_{c}\right)$, the light cone results on an Arago spot on the glass/liquid interface. This spot is totally reflected on the interface and leads to an out running evanescent wave. The evanescent field corresponds to a disk with a radius $\delta_{G-H}$. b) For a higher angle of incidence, the Arago spot is within the glass and the evanescent wave on the interface has an annular shape that is centered on the trap position. The evanescent wave holds on a smaller distance since the Goos Hänchen effect is smaller, and corresponds to a donut.

an angle of incidence $\tan i=d / 2 e$, that constitute the evanescent field on the interface, $\delta_{G-H}$ is smaller. Then the donut shape of the electric field is reduced compared with a smaller diffracting disk.

Consequently, on the glass/liquid interface, in the liquid medium, the electric field is essentially composed of out running evanescent waves centered on the Arago spot, leading to an electromagnetic field with a donut distribution. This donut gets smaller when increasing the size of the diffracting beam. Similar donut shape evanescent waves can be obtained using an occulted beam focused by a high numerical objective [35]. However, in that experiment, the optical beam results from a focusing and its size is thus limited by diffraction. It corresponds to a size of the order of a wavelength, whereas in our experiment, the beam size can be smaller [58].

\section{RESULTS}

We have followed the trapping of more than 250 particles and registered the lifetime of the trapping $\tau$ versus the velocity flow (see Fig. 3), for a diffracting diameter disk of $d=650 \mu \mathrm{m}$. Most of the results except those on the study of the disk diameter have been obtained with this disk diameter, since it is easier to trap particles with a larger disk. This velocity flow has been estimated from the velocity of nearby particles that are not trapped. The slower the velocity the longer the trap lasts. The variation of $\tau$ is linear with the flow velocity. For a nearly Brownian motion of the particles, $\tau$ can reach $0.8 \mathrm{~s}$. We will come back later on this linear behavior (see section IV B).

An example of the fluorescence of a trapped particle is displayed in Fig. 4a. The circle is a guide for the eye. We have registered the intensity of the fluorescence versus time before, during and after the trapping time (see Fig. 4b). Clearly, and surprisingly, during the trapping period, the fluorescence decreases by a factor of 3 . This is a clear signature of the trapping time. However, the other side of the coin, is that during the trapping time there is much less fluorescence and we may not detect the particles any more. We will return to the fluorescence 

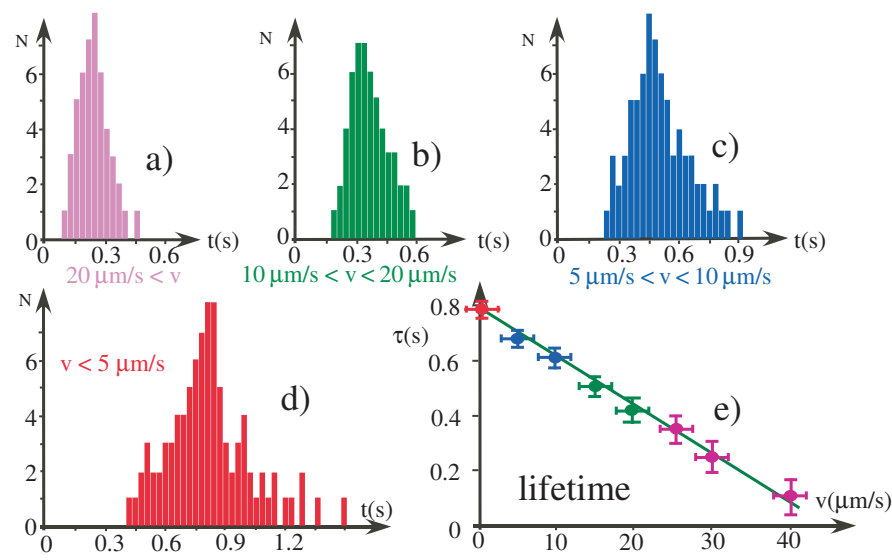

FIG. 3. Trap lifetime. a), b), c), d) Histogram of the trapping time for different flow velocities. $N$ : number of particles that have been trapped. e) Variation of the mean trapping lifetime versus flow velocities. Crosses: experimental data (the size of the cross represents the measurement uncertainty), straight line: linear adjustment.
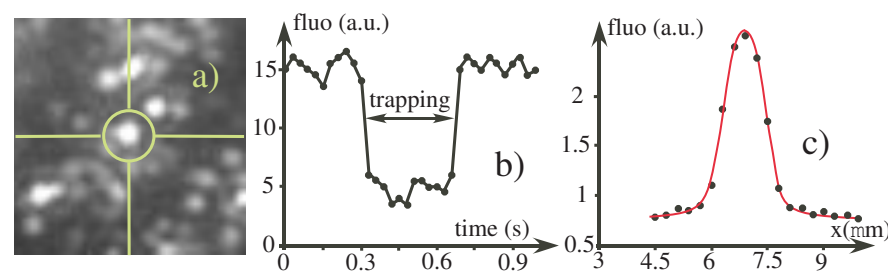

FIG. 4. a) Example of the fluorescent signal from a trapped particle. It lies at the center of the circle that used as a guide for the eye. b) Variation of the fluorescent signal versus time. The decrease of the signal corresponds to a trapping time. c) Detected signal of a particle versus position. Each point corresponds to a single pixel signal. Solid line: Gaussian fit that enables to register the center of the particle position with a $10 \mathrm{~nm}$ precision.

decrease in the next section (sect. IV). The detected fluorescence of the trapped particles corresponds to several pixels on the camera (one pixel corresponds to $300 \mathrm{~nm}$ ). However, we can perform a Gaussian adjustment of the signal and assimilate the center of the Gaussian fit with the position of the center of the particle with an accuracy better than $10 \mathrm{~nm}[61,62]$. Such an example of the fluorescence signal is displayed in Fig. 4c.

We can thus follow the trajectory of the particle before, during, and after the trapping. In particular, we can register the position of the center of the particle during a trapping time. An example of such a trajectory appears in Fig. 5. We can thus determine the diameter of the trap. We have also varied the diameter $d$ of the diffracting chromium disk an estimated the trap radius $r_{t}$. It equals $r_{t}=125,62$, and $50 \mathrm{~nm}$ for $d=650,700$, and $750 \mu \mathrm{m}$, respectively. We will discuss this result in the next section (section IV C). We were not able to trap particles with diffracting disks of higher diameters, probably because the intensity of the Arago spot was too low.

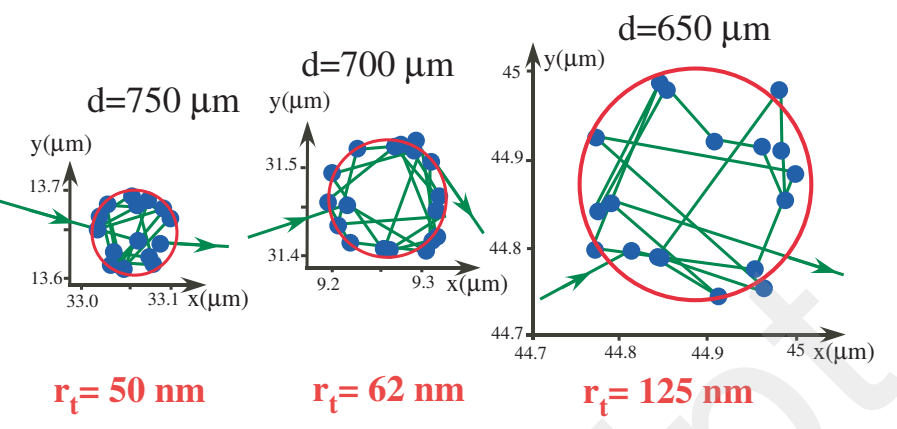

FIG. 5. Position of the center of the particle during the trapping period for chromium disks of diameter 750, 700, and 650 $\mu \mathrm{m}$ respectively. The trap size decreases when the size of the disk increases. The position of the particle is registered every $30 \mathrm{~ms}$. The diameter of the particle is $200 \mathrm{~nm}$, i.e. bigger than the trap size what ever the size of the radius of the trap $r_{t}$.

The intensity of the spot varies as $1 / d^{2}$, according to eq. 1 .

\section{DISCUSSION}

The dipole force and the resulting potential exerted on a dielectric sphere are well known and have been calculated several times. Let us recall its expression. The potential writes $[4,52,63,64]$

$$
V_{d i p}(r)=-\frac{2 \pi a^{3}}{c} \frac{\rho^{2}-1}{\rho^{2}+2} n_{0} I(r)
$$

where $c$ is the velocity of light, $\rho$ is the ratio between the optical index of the sphere and the liquid respectively $\left(\rho=n_{0} / n_{l}=1.14\right)$, and $I(r)$ is the light intensity distribution. In order to determine the force, one has only to replace $I(r)$ by its derivative $(\nabla I(r))$, since the force derives from the potential. As already mentioned, the maximum intensity is $I(0)=40 \mathrm{~mW} \cdot \mathrm{mm}^{-2}$, the beam size is $120 \mathrm{~nm}$, leading to a potential depth $V_{d i p}=1.1 \times 10^{-25}$ $\mathrm{J}$, and a trapping force of the order $F_{d i p}=9.3 \times 10^{-19}$ $\mathrm{N}$. This is the force just at the interface but, due to the penetration depth of the evanescent wave, the force and the potential are even fainter.

Compared with the thermal energy $E_{t h}=k_{B} T\left(k_{B}\right.$ being the Boltzmann constant) and for a temperature of the order of $T=300 \mathrm{~K}$ which leads to $E_{t h} \simeq 4 \times$ $10^{-21} \mathrm{~J}$. Taking into account some heating due to the light interaction would lead to even higher energies. This potential is really too low to trap any particle with a radius in the hundred of nanometer range. The dipole force may play a role with higher diameter particles using smaller disks. In that case, even non-fluorescent particles (in the micrometer diameter range) could be trapped, as for usual optical tweezers. However we are interested here in the trapping of nanometer particles. One may then wonder what could be the trapping mechanism? 


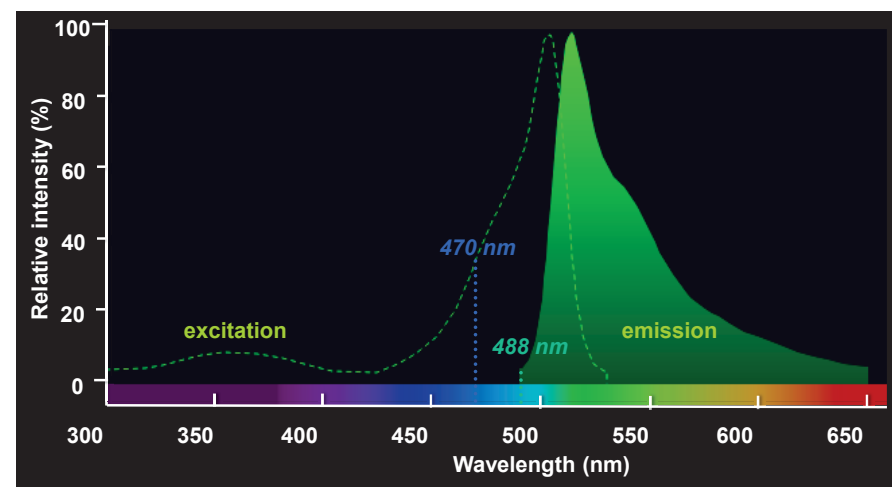

FIG. 6. Excitation spectrum (dotted line) and fluorescence spectrum (solid line) of the particles (adapted from [67]).

In our trapping experimental set-up, the Arago spot with sizes below the wavelength, may resemble so-called super oscillating beams. Such super-oscillating beams have already trapped small particles $[65,66]$. However, the trapping mechanism they used is based on the dipolar force. As already mentioned, it strongly decreases with the trapped particle size. Besides, these super-oscillating beams also generate side lobes very close to the fundamental lobe. These side lobes are much more intense than the fundamental one. Consequently, for very small beams, particles may be trapped in the side lobe instead of the fundamental one. This may be a limit to reaching small trap sizes. Moreover, whereas the trapping mechanism is very efficient in $2 \mathrm{D}$, it is less efficient in the direction of the focusing of the beam. The trapping dimension in this direction is in the micrometer range, as for optical tweezers. The question of the trapping mechanism in our case is still unsolved.

\section{A. Origin of the trapping force}

We have already mentioned that the trapping mechanism relies on stimulated emission of the excited nanoparticle in the evanescent waves [54]. Let us detail it. The excitation and fluorescence spectra appear in Fig. 6. The principle of the trapping is described in Fig. 7. The particle is excited by the diode at $\lambda=470 \mathrm{~nm}$. It then de-excites by emitting a photon in a stimulated way in the evanescent waves. Since the evanescent waves are outgoing waves in our configuration, the particle gets pushed towards the center of the Arago spot. To support this hypothesis, we have switched the wavelength of the evanescent wave from $\lambda=488 \mathrm{~nm}$ to $\lambda=404 \mathrm{~nm}$ with exactly the same configuration. This wavelength is out of the fluorescence spectra of the particle. The mean wavelength of the diode responsible for the excitation of the particle is still $\lambda=470 \mathrm{~nm}$. We notice that there is no more particle trapped in this case.

As already mentioned, we have registered the fluorescence from the particle before, during and after the trap-

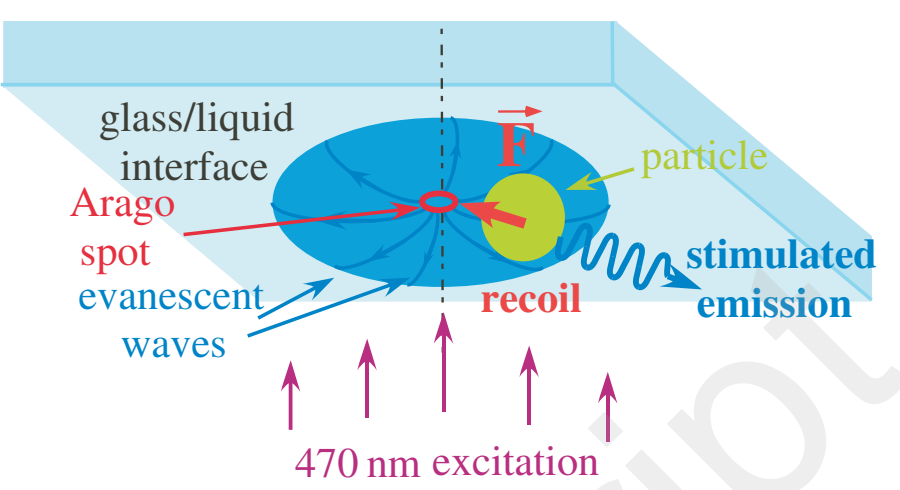

FIG. 7. Principle of the trapping mechanism. The particle is pushed towards the upper side of the channel by the radiation pressure from the $470 \mathrm{~nm}$ diode that excites the particle (beam at the bottom of the drawing). Then the particle reemits light in a stimulated way at $488 \mathrm{~nm}$ in the outgoing evanescent wave. The particle is thus pushed towards the center of the trap by the force $F$.

ping period (see Fig. 4c). There is a decrease of the fluorescence signal by a factor of three during the trapping time. It then recovers its initial fluorescent rate when leaving the trap. Since the radiation power from the 470 $\mathrm{nm}$ diode is unchanged there must be a coherent process that de-excites the particle. It is very similar to the selective deactivation of fluorophores in STED microscopy $[68,69]$. It means that the fluorescent particle gets deexcited via stimulated emission instead of spontaneous emission. Other quenching mechanisms [70] are unlikely in our case, since the fluorescence of the particle recovers its initial value after trapping. This is a signature of the stimulated emission process.

Let us focalized in the order of magnitude of the forces linked to the stimulated emission that lies in the horizontal plane, and also to the force in the vertical direction. Concerning this last force, the light at the wavelength $\lambda=470 \mathrm{~nm}$ plays a central role in the trapping. Indeed, each fluorescent particle within the flow interacts with this field. In a fluorescent cycle, the particle absorbs a photon at $\lambda=470 \mathrm{~nm}$ and gets excited. Besides, since light carries linear momentum, due to momentum conservation, the particle recoils towards the direction of the light impulsion, i. e. towards the glass/liquid interface. This radiation pressure force is estimated to $F_{R}=n_{l} \hbar k \Gamma=3.7 \times 10^{-14} \mathrm{~N}$, where $\hbar$ is the reduced Planck constant. It acts on all the particles all along the channel, which therefore flow just underneath the glass/liquid interface. The electrostatic repulsion prevents them to stick to the glass, and keeps them away at a fraction of hundreds of nanometer distance [71, 72]. Thereafter, particles fluoresce and recoil in a random direction when not trapped.

In order to evaluate the forces in the other directions, let us develop the model already used previously [54] and detail the calculations. In the horizontal plane, the force 
is due to stimulated emission in the evanescent wave. To estimate the value of the force, we evaluate the number of stimulated photons emitted per second. This can be performed via evolution equations [73]. We assume here a simplified two level system with one excited state and one ground state. The population in the excited $n_{e}$ is fed by the pumping diode at $\lambda=470 \mathrm{~nm}$. It is proportional to the population in the ground state $n_{g}$, and to the spectral density of energy of light at $\lambda=470 \mathrm{~nm}, \rho\left(\nu_{0}\right)$. It writes $d n_{e} / d t=B n_{g} \rho\left(\nu_{0}\right)$, where $B$ is the so-called Einstein coefficient [74]. The decrease arises from several terms. One is due to spontaneous emission. This term writes $d n_{e} / d t=-A n_{e}$, where $\mathrm{A}$ is the Einstein coefficient linked to spontaneous emission. An other term is associated to the stimulated emission and writes $d n_{e} / d t=-B n_{e} \rho(\nu)$, $\rho(\nu)$ stands for the spectral density of energy of light at $\lambda=488 \mathrm{~nm}$. We also add a last term $d n_{e} / d t=-C n_{e}$ due to non radiative decay, where $\mathrm{C}$ is the associated decay factor. Then, at equilibrium, when a steady state situation is reached, the balancing between absorption and emission writes

$$
A n_{e}+B n_{e} \rho(\nu)-B n_{g} \rho\left(\nu_{0}\right)+C n_{e}=0
$$

In the absence of the $\lambda=470 \mathrm{~nm}$ radiation, eq. 3 simplifies to

$$
A n_{e}^{0}-B n_{g}^{0} \rho\left(\nu_{0}\right)+C n_{e}^{0}=0
$$

where the superscript 0 accounts for the absence of the Arago spot. Let us call $\delta=\rho(\nu) / \rho\left(\nu_{0}\right)$ the ratio between the spectral energy density at $488 \mathrm{~nm}$ and $470 \mathrm{~nm}$, respectively. Let us call $\alpha=n_{e} / n_{e}^{0}$ the ratio between the populations in the excited state with and without the Arago spot. Since $n_{e}+n_{g}=1$ and $n_{e}^{0}+n_{g}^{0}=1$, we can replace $n_{e}=\alpha n_{e}^{0}, n_{g}=1-\alpha n_{e}^{0}$, and $n_{g}^{0}=1-n_{e}^{0}$. Then, multiplying eq. 4 by $\alpha$ and subtracting it from eq. 3, leads to $n_{e}^{0}=(1-\alpha) /(\alpha \delta)$.

We can evaluate the value of $n_{e}^{0}$ from our experimental optical parameters. Indeed, $n_{e}^{0}=s /(1+s), s$ being the saturation parameter. For a saturation that goes to infinity, $n_{e}^{0}=1 / 2$, and for $s=2$, which corresponds to the experimental situation, $n_{e}^{0}=1 / 3$. Besides, the power of the diode at $470 \mathrm{~nm}$ is $20 \mathrm{~mW}$. It is focused to a waist of $1 \mathrm{~mm}^{2}$, leading to an intensity of $6 \mathrm{~mW} / \mathrm{mm}^{2}$ and a value of $\delta=6$. The value of $\alpha$ is then $\alpha=1 / 3$ which is approximately the value deduced from Fig. $4 \mathrm{~b}$.

The decay of the population in the excited state due to stimulated emission can be linked to the increase of the population due to absorption in the absence of the Arago spot $B n_{e} \rho(\nu)=B \alpha n_{e}^{0} \delta \rho\left(\nu_{0}\right)$. Since $n_{e}^{0}=1 / 3, n_{g}^{0}=$ $2 / 3$ and $n_{e}^{0}=n_{g}^{0} / 2$, this leads to $B n_{e} \rho(\nu)=B n_{g}^{0} \rho\left(\nu_{0}\right)$. $B n_{e} \rho(\nu)$ is the decrease of the population of the excited state due to stimulated emission. It is proportional to the stimulated force. The term $B n_{g}^{0} \rho\left(\nu_{0}\right)$ is the increase of the population of the excited state due to absorption. It is proportional to the radiation pressure force. Then, the force due to stimulated emission $F$ equals the force due to the radiation pressure $F_{R}$ in the absence of the
Arago spot, since the two wavelengths are close. This leads to $F=3.7 \times 10^{-14} \mathrm{~N}$.

It is worth noting that the force from the $\lambda=470$ nm diode is then the sum of the term due to "absorption followed by the spontaneous emission process" that has decreased by a factor of three (decrease of the spontaneous emission) and the term due to "absorption followed by a stimulated emission process". This force is then $F_{T}=F_{R} / 3+F=4.93 \times 10^{-14} \mathrm{~N}$. During the trapping period, the force pushing the particle towards the interface is more effective, since the number of photon absorption per second is increased, compared with the non trapping time.

This value of the force due to stimulated emission can be estimated experimentally. For example, for a diffracting disk of a diameter of $650 \mu \mathrm{m}$, the radius of the trap, according to Fig. 5c, is $125 \mathrm{~nm}$. Due to heating, the temperature increase, compared with the situation without laser, is estimated roughly to $30 \mathrm{~K}$ (see below). Then $T=323 \mathrm{~K}$, leading to a thermal agitation $1 / 2 k_{B} T=2.2 \times 10^{-21} \mathrm{~J}$. Then, from the equipartition theorem, one can estimate the trap stiffness $\left.\kappa=k_{B} T /<x^{2}\right\rangle=2.85 \times 10^{-7} \mathrm{Nm}^{-1}$. The force is then $F=\kappa \times \sqrt{\left\langle x^{2}\right\rangle}=3.6 \times 10^{-14} \mathrm{~N}$, which is nearly the value we estimate numerically. This reinforces the calculation of the force we deduced from equations of the populations (eqs. 3 and 4 ).

\section{B. Trap lifetime}

From the following of the trapping of the 250 particles (see Fig. 3), we have plotted the mean trap lifetime $\tau$ versus the liquid flow velocity (see Fig. 3e). As already mentioned, the lifetime $\tau$ decreases linearly with the liquid flow velocity. It reaches $0.8 \mathrm{~s}$ at most for a Brownian motion of the particles. One can link theoretically the lifetime $\tau$ and the flow velocity by identifying the different potentials and energies. On the one hand, the trapping potential $U_{0}$ can be associated to the work of the force. It is thus equal to the force times the typical radius of the trap $\left(r_{t}=125 \mathrm{~nm}\right.$ for $\left.d=650 \mu \mathrm{m}\right)$. Although it is not absolutely correct, and for the sake of simplicity, we assume here that the trap is isotropic. This leads to $U_{0}=6.2 \times 10^{-21} \mathrm{~J}$.

On the other hand, some elements tend to push the particle outside the trap. First, thermal agitation is one escape term. It has already been calculated in the preceding section and equals $3 / 2 k_{B} T=6.7 \times 10^{-21} \mathrm{~J}$. The second one comes from with the energy associated with friction force with the flow $\gamma v r_{t}, v$ being the flow velocity, and $\gamma=6 \pi a \mu, \mu$ the dynamical viscosity. This force acts in the direction of the flow only. At $323 \mathrm{~K}$,

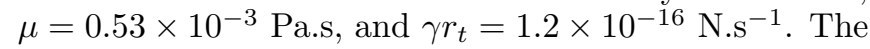
last term is the diffusion in momentum space [75], due to the random recoil following an absorption-spontaneous emission cycle. It equals to $D=\hbar^{2} k^{2} \Gamma / 3=1.3 \times 10^{-41}$ J.kg.s ${ }^{-1}$. It is divided by 3 since during the trapping 


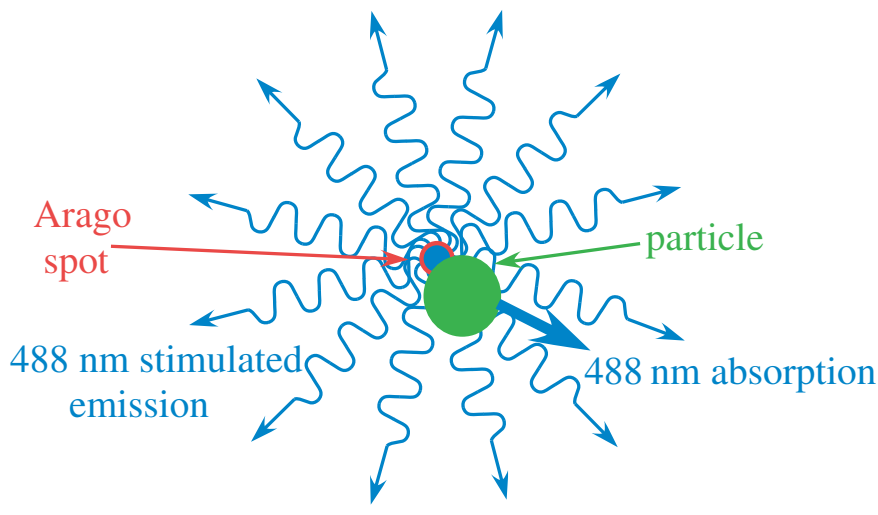

FIG. 8. The particle can absorb light at $488 \mathrm{~nm}$ and re-emit it at the same wavelength in another direction. This leads to a redistribution of the photons among the various directions and increases significantly the diffusion process.

period, the fluorescence is divided by 3 . Note that this momentum diffusion coefficient is different from the usual position diffusion coefficient defined by Einstein $D_{e}=k_{B} T / \gamma$. Indeed, the Einstein coefficient is relevant for a diffusion process in position, whereas, in order to estimate the trapping time, we have to consider the diffusion in momentum space.

The following equation then sets

$$
U_{0}=\frac{3}{2} k_{B} T+\gamma v r_{t}+\frac{1}{m} D \tau,
$$

$m$ being the mass of the particle. One expects a linear variation of $\tau$ versus the flow velocity, as experimentally observed (see Fig. 3c). Let us have a closer look. As the flux velocity tends towards zero, $\tau=0.8 \mathrm{~s}$. The term associated with the diffusion equals $2.4 \times 10^{-24} \mathrm{~J}$. This value is too low, since the thermal agitation and the potential are in the $10^{-21} \mathrm{~J}$ range.

There is still one problem that must be addressed (see Fig. 3c). In this figure, the value $v=45 \mu \mathrm{m} . \mathrm{s}^{-1}$ corresponds to $\tau=0$. The energy associated with the friction term then equals $\gamma v r_{t}=5.6 \times 10^{-21} \mathrm{~J}$. This is of the same order of magnitude as the other terms in Eq. 5. It has to be noted that this energy is relative to the flow around the particle. However, the flow velocity $v$ is estimated from the fluorescent particles around the trapped particle. Nevertheless, this trapped particle is close to the interface. Indeed, as already mentioned, the radiation pressure force from the diode at $\lambda=470 \mathrm{~nm}$ is higher during the trapping time than in a non trapping time. The particle must be closer to the interface. The particle velocity must decrease while approaching the surface. For example, in a Poiseuille flow in a channel of $30 \mu \mathrm{m}$ height, the velocity from a distance of $1.5 \mu \mathrm{m}$ from the interface (that is a raisonable distance for the particles to travel along the channel) to a distance of $100 \mathrm{~nm}$ (that is the distance of the particles from the interface while trapped) decreases by a factor of 10 . The flow velocity in Fig. $3 \mathrm{c}$ is thus overestimated by a factor of around $10 \mathrm{in}$ that case. Then, the experimental results agree with the model described by Eq. 5 taking into account a velocity correction in the term $\gamma v r_{t}$.

Actually, there is another diffusion process. The stimulated emission wavelength $(\lambda=488 \mathrm{~nm})$ lies within the absorption spectrum of the particle. Then, there must be absorption-stimulated-emission cycles at this precise wavelength. These cycles are difficult to evaluate. Since, the wavevectors are directed radially from the center, there might be absorption in one direction and emission in a stimulated way in another direction. The photons are redistributed among the various possible directions (see Fig. 8) This dramatically increases the diffusion process when the particle is trapped. In order to reduce it, one solution could be to use an Arago spot at a higher wavelength but still in the emission spectra of the dye molecules, thus preventing from re-absorption.

\section{Trap size}

The trap size and the behavior of the particle inside the trap are important issues within the framework of manipulating them. Up to now, we have mainly discussed the results obtained with the $125 \mathrm{~nm}$ radius trap due to the diffraction of light with a $650 \mu \mathrm{m}$ occulting disk. However, as presented in Fig. 5, as the diffracting disk gets bigger, the trap size diminishes. Actually, the Arago spot size diminishes, and the angle of incidence of the rays forming the spot increases. Then, the Goos-Hänchen effect and the spreading of the evanescent wave get smaller. From our model, the trapping mechanism corresponds to stimulated emission in the evanescent wave. The trap size should depend on the Goos-Hänchen effect. The longitudinal shift along the interface for a linear polarization writes [76]

$$
\delta_{G-H}=\frac{\lambda}{\pi} \frac{\sin i \cos i}{\sqrt{n_{g}^{2} \sin ^{2} i-n_{l}^{2}}}
$$

This Goos-Hänchen shift is displayed in Fig. 9 together with the trap size, according to Fig. 5. It turns out that the trap size varies linearly with the shift, as expected.

Let us have a closer look at the particle position inside the trap that still needs to be addressed (see Fig. 5). Whatever the trap size, the particle position lies at the edges of the trap, and hardly at the center. However, one has to remind that the trap radii are 125, 62 and $50 \mathrm{~nm}$, respectively, depending on the diffracting disk radius and that the particle radius is $a=100 \mathrm{~nm}, \mathrm{i}$. e. of the same order of magnitude as the trap dimensions. The concept of the particle position within the trap might be difficult to conceive. Nevertheless, let us evaluate the damping ratio as for the harmonic oscillator. It is the ratio of the damping coefficient (here $\gamma / 2 m$ ) with the resonant pulsation $\left(\omega_{0}=\sqrt{\kappa / m}\right)$. The damping ratio is then $\gamma /(2 \sqrt{m \kappa})=445$. Although the trap is over damped, the particle spends little time at the center of the trap. 


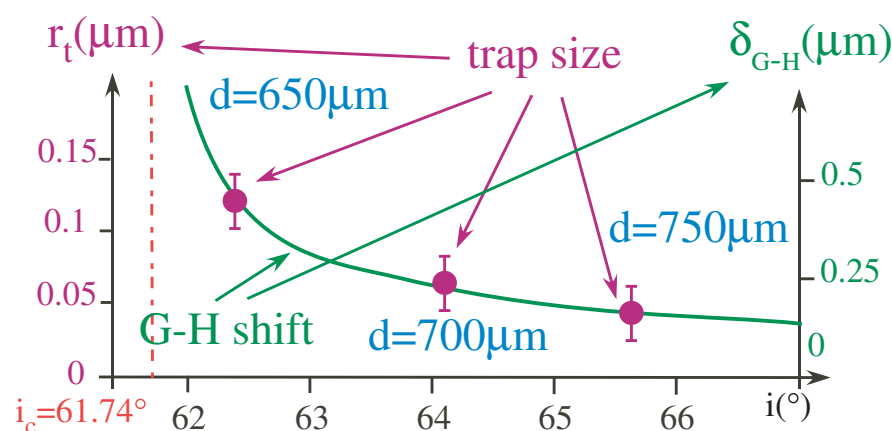

FIG. 9. Points: size of the trap according to figure 5 versus angle of incidence corresponding to the size of the chromium disk. The error bars are estimated from several trapped particle trajectories and from the uncertainty of the measure of the center size. Solid line: theoretical Goos-Hänchen (G-H) shift versus angle of incidence.

There may be different reasons for that. First, as already explained, the diffusion due to stimulated emission within the components of the evanescent wave, is much higher at the center of the trap than on the edges, leading to a drift force from the center.

There might be another reason linked to temperature gradients and self thermophoresis [77-79]. In an absorption (mean wavelength $\lambda_{1}=470 \mathrm{~nm}$ ) spontaneous emission (mean wavelength $\lambda_{2}=550 \mathrm{~nm}$ for the considered fluorescent particles) cycle, the energy difference between the two wavelengths is $\Delta E=h c \times\left(1 / \lambda_{1}-1 / \lambda_{2}\right)=$ $6.1 \times 10^{-20} \mathrm{~J}$. The number of cycles for a trapped particle equals $\Gamma / 3$ (see sect. IV A). This generates heat. The power to be evacuated is of the order of $P=\Delta E \times \Gamma / 3 \simeq$ $4 \times 10^{-7} \mathrm{~W}$. For a trapping region of radius $r_{t}=125 \mathrm{~nm}$, the heat flux equation [80] across the surface $S$ limiting the trapping volume ( $S=4 \pi r_{t}^{2}$, spherical symmetry), leads to a temperature gradient $\frac{d T}{d r}=\frac{P}{S k} \simeq 3 \times 10^{6}$ $\mathrm{K} . \mathrm{m}^{-1}, k=0.6 \mathrm{~W} . \mathrm{m}^{-1} . \mathrm{K}^{-1}$ being the thermal conductivity of water and $r$ the radial distance. We assume here a steady state flux for a mean particle position at the center of the trap. The drift velocity [81] then equals $u=D_{T} \frac{d T}{d r} \simeq 3 \mu \mathrm{m} . \mathrm{s}^{-1}, D_{T}=10^{-8} \mathrm{~cm}^{2} . \mathrm{s}^{-1} \cdot \mathrm{K}^{-1}$ being the thermophoretic mobility. This drift velocity decreases as the particle leaves the center of the trap.

The order of magnitude of the velocity drift in the vicinity of the center of the trap is around $1 \mu \mathrm{m} . \mathrm{s}^{-1}$, i.e., of the same magnitude as the flow velocity. This may prevent the particle to lie in the trap center. This is precisely what we observe (see Fig. 5). Although the radius of the trap is very small, if the thermophoresis hypothesis is correct, this drift may one drawback of using fluorescent particles. This may also be a limiting factor of the measured lifetime. It is worth noting that similar position distributions have been reported in the trapping of fluorescent particles, out of equilibrium [82-84]. However these particles were Janus particles and the direction of the thermophoretic force is oriented with their dissymmetry. In our case, the explanation is different since we deal with spherical particles.

\section{OUTLOOKS}

In our experiment, the trapping potential is nearly twice the thermal agitation. To be efficient, the trapping potential should be around 10 times the thermal agitation [1]. Besides, we have identified another detrimental factor that is the diffusion due to stimulated emission redistribution among the evanescent wave directions. This limits the trap lifetime to $0.8 \mathrm{~s}$ at most, which is too low for the usual applications of optical traps.

This lifetime can be increased using two complementary ways. First, an increase of the $488 \mathrm{~nm}$ laser power by a factor of 10 would increase the intensity of the evanescent wave by a factor of 10 without reaching the damage threshold of the particles. Compared with the present experiment, the light intensity would then be of the order of $400 \mathrm{~mW} \cdot \mathrm{mm}^{-2}$. The stimulated emission and thus the trapping force in the horizontal directions would increase accordingly. Moreover, an increased of the $488 \mathrm{~nm}$ laser power will thereby increase the stimulation emission process. It will thus reduce the population of the excited state of the fluorescent particles. It will also increase the radiation pressure force and the trapping in the vertical direction, and will thereby lower the momentum diffusion due to spontaneous emission.

Second, one can switch the wavelength of the Arago spot to a higher wavelength (typically in the green region of the optical spectrum, between 500 and $560 \mathrm{~nm}$, which is still in the emission spectrum of the dye molecules). This will lower the evanescent wave reabsorption, and then also lower the diffusion due to stimulated emission redistribution, which is probably the main limiting factor of the trap lifetime.

Such trapping of a single particle could find applications in the pattern construction of small particles deposited on a substrate [85-87]. For example, while a particle is trapped, a laser pulse could be sent also via the optical fiber that pushes the particle towards the substrate or induces a chemical reaction with the substrate. The particle would be glued on it. Then, thanks to the microstage, the spot could be moved and accurately positioned at another place, another particle could be trapped and glued, and so on. One can thus print a regular pattern of glued particles on the substrate.

One of the characteristics of this trapping technique compared with techniques using gradient forces, is that the laser intensity is rather low (in the $10 \mathrm{~mW} \cdot \mathrm{mm}^{-2}$ range) [88]. One can then consider the manipulation of nano-objects inside living organisms below photodamage. Besides, the trap position can be moved by sliding the occulting disk on the channel using microstages, in a way analogous to the move of an immersion objective in a microscope. The experiment could be easily transposed to any wavelength, including infrared, depending on the transparency window of the experiment. The type of flu- 
orescent particles has then to be adjusted. For example, such trapping system in the surface-plane could be used to trap fluorophores attached to organelles, viruses or even proteins in a lab-on-a-chip integration. The typical size of conventional optical tweezers is too big to trap them efficiently. It could then serve in transfection and single-and sub-cellular surgery [89] or for single-cell biopsies [90], and be used as a light nanorobot [91, 92].

However, the most promising application of this trapping is foreseen in the trapping of even smaller particles. As the diameter of the particles is reduced, the number of fluorescent molecules in the particles will be reduced, the fluorescence duty cycle and the force will be reduced accordingly. However, the relevant quantity is the acceleration of the particle, and its mass will diminish with the same ratio as the force. The acceleration will be unchanged. What could be the limit for the size of the trapped particles. Considering eq. 5, the diffusion term is small regarded to the potential. The increase of stimulated emission will lead to a decrease of this term. The diffusion due to redistribution of photons among the evanescent waves decreases because of the use of a higher wavelength. The most bothering and limiting term would then be the thermal agitation term. By increasing the laser power, one may consider trapping particles with a 10 times smaller radius (i. e. in the $10 \mathrm{~nm}$ range), but not below.

Nevertheless, the trapping mechanism evoked here, is borrowed from laser trapping techniques [93]. The cold atom community usually deals with gases. In order to trap atoms or molecules, they only consider those with already low thermal agitation with initial temperatures around $10 \mathrm{~K}$ or below, or atoms or molecules in the tail of the Maxwell-Boltzmann distribution of the gas. Traps using the dipolar force for atoms have already been reported [94, 95]. Besides, traps in the vicinity of surfaces have been considered [96]. One could then imagine trapping several atoms or even molecules in a very small volume (much smaller than usual for optical traps) close to a surface. In this later case, the experiment would hold in vacuum and the optical transitions of the atoms or molecules would replace the transition of the dye molecules in the particle. It could thus be an alternative route towards Bose-Einstein condensation [97, 98] using a smaller number of atoms or molecules in a much smaller volume.

\section{CONCLUSION}

To conclude, we have detailed a new optical trap for single fluorescent particle with 100 nanometer radius. The trapping mechanism is based on stimulated emission in the evanescence on a totally reflected Arago spot diffracted from a chromium disk. The smallest trap we observe has a radius of $50 \mathrm{~nm}$. In the other direction the trapping mechanism is the radiation pressure force that pushes the particle towards the interface. The longest lifetime we observe is $0.8 \mathrm{~s}$, and is limited, on the one hand, by the liquid flux around the particle and by the thermal diffusion, and, on the other hand, by stimulated emission redistribution of the photons in the evanescent wave from the Arago spot.

Since the optical intensity is low (in the $10 \mathrm{~mW} \cdot \mathrm{mm}^{-2}$ range) compared with other trapping techniques, the trapping of small living cells such as virus and organelles can be considered. This could have valuable applications in biomedical manipulations. Pushing the limits of the trap size and the particles down the nanometer range and below, the trapping mechanism described here could offer new techniques in cold atoms and cold molecules manipulation. In particular, the trap size could reach dimensions smaller than $100 \mathrm{~nm}$, that is much smaller than the dimensions usually obtained in optical traps for cold atoms that are in the hundred of $\mu \mathrm{m}$ range.

\section{ACKNOWLEDMENT}

The authors would like to thank Dr. Hervé TABUTEAU for valuable discussions. We acknowledge support from the Contrat Plan Etat Région CPER BUFFON.

\section{AUTHORS CONTRIBUTIONS}

All the authors were involved in the preparation of the manuscript and contribute equally. All the authors have read and approved the final manuscript.
[1] A. Ashkin, J. M. Dziedzic, J. E. Bjorkholm, and S. Chu, Opt. Lett. 11 (1986) 288.

[2] A. Ashkin, IEEE J. Sel. Top. Quantum Electron. 6 (2000) 841.

[3] D. G. Grier, Nature 424 (2003) 810.

[4] K. C. Neuman and S. M. Block, Rev. Sci. Instrum. 75 (2004) 2787.

[5] O. M. Maragò, P. H. Jones, P. G. Gucciardi, G. Volpe, and A. C. Ferrari, Nat. Nanotech. 8 (2013) 807.
[6] A. Gennerich, Optical Tweezers. (Springer, New York, 2017).

[7] P. Polimeno, A. Magazzù, M. A. Iatì, F. Patti, R. Saija, C. D. E. Boschi, M. G. Donato, P. G. Gucciardi, P. H. Jones, G. Volpe, and O. M. Maragò, J. Quant. Spectrosc. Radiat. Transfer 218 (2018) 131.

[8] R. Bowman, G. Gibson, and M. Padgett, Opt. Express 18 (2010) 11785. 
[9] E. Bertseva, D. Grebenkov, P. Schmidhauser, S. Gribkova, S. Jeney, and L. Forró, Eur. Phys. J. E 35 (2012) 1.

[10] G. Sancataldo, L. Scipioni, T. Ravasenga, L. Lanzanò, A. Diaspro, A. Barberis, and M. Duocastella, Optica 4 (2017) 367.

[11] U. F. Keyser, J. Van der Does, C. Dekker, and N. H. Dekker, Rev. Sci. Instrum. 77 (2006) 105105.

[12] D. B. Phillips, S. H. Simpson, J. A. Grieve, R. Bowman, G. M. Gibson, M. J. Padgett, J. G. Rarity, S. Hanna, M. J. Miles and D. M. Carberry, EPL 99 (2012) 58004.

[13] F. Qian, S. Ermilov, D. Murdock, W. E. Brownell, and B. Anvari, Rev. Sci. Instrum. 75 (2004) 2937.

[14] P. Mangeol, D. Côte, T. Bizebard, O. Legrand, and U. Bockelmann, Eur. Phys. J. E 19 (2006) 311.

[15] G. V. Soni, M. P. Jonsson, and C. Dekker, Small 9 (2013) 679.

[16] X. Wang, S. Chen, M. Kong, Z. Wang, K. D. Costa, R. A. Li, and D. Sun, Lab Chip 11 (2011) 3656.

[17] C. W. Shields IV, C. D. Reyes, G. P. López, Lab Chip 15 (2015) 1230.

[18] A. Keloth, O. Anderson, D. Risbridger, L. Paterson, Micromachines 9 (2018) 434.

[19] M. C. Noom, B. Van Den Broek, J. Van Mameren, and G. J. Wuite, Nat. Methods 4 (2007) 1031.

[20] B. Jagannathan, and S. Marqusee, Biopolymers 99 (2013) 860.

[21] B. M. Mihiretie, P. Snabre, J.C. Loudet, and B. Pouligny, Eur. Phys. J. E 37 (2014) 124.

[22] J. A. Rodrigo, and T. Alieva, Optica 2 (2015) 812.

[23] Z. Zhang, T. E. Kimkes, and M. Heinemann, Sci. Rep. 9 (2019) 19086.

[24] R. Zhu, T. Avsievich, A. Popov, and I. Meglinski, Cells 9 (2020) 545.

[25] M. Dienerowitz, M. Mazilu, and K. Dholakia, J. of Nanophoton. 2 (2008) 021875.

[26] D. Gao, W. Ding, M. Nieto-Vesperinas, X. Ding, M. Rahman, T. Zhang, C. T. Lim, and C. W. Qiu, Light Sci. Appl. 6 (2017) e17039.

[27] J. E. Melzer, and E. McLeod, ACS Nano 12 (2018) 2440.

[28] D. G. Kotsifaki, and S. N. Chormaic, Nanophotonics 8 (2019) 1227.

[29] D. Choudhary, A. Mossa, M. Jadhav, and C. Cecconi, Biomolecules 9 (2019) 23.

[30] D. G. Grier, Nature. 424 (2003) 810.

[31] M. Daly, M. Sergides, and N. S. Chormaic, Laser Photon. Rev. 9 (2015) 309.

[32] P. A. Maia Neto, and H. M. Nussenzweig, EPL 50 (2000) 702 .

[33] S. E. S Spesyvtseva, and K. Dholakia, ACS Photonics 3 (2016) 719.

[34] E. Almaas, and I. Brevik, J. Opt. Soc. Am. B 12 (1995) 2429.

[35] M. Gu, J.-B. Haumonte, Y. Micheau, J. W. N. Chon, and X. Gan, Appl. Phys. Lett. 84 (2004) 4236.

[36] D. Ganic, X. Gan, and M. Gu Opt. Express 12 (2004) 5533.

[37] Y. Z. Yoon, and P. Cicuta, Opt. Express 18 (2010) 7076.

[38] Y. Xiang, X. Tang, C. Min, G. Rui, Y. Kuai, F. Lu, P. Wanf, H. Ming, Q. Zhan, X. Yuan, J. R. Lakowicz, and D. Zhang, Ann. Phys. 532 (2020) 1900497.

[39] S. E. Skelton, M. Sergides, R. Patel, E Karczewska, O. M. Maragó, and P. H. Jones, J. Quant. Spectrosc. Radiat. Transfer 118 (2012) 2512.
[40] M. C. Frawley, I. Gusachenko, V. G. Truong, M. Sergides, and S. N. Chormaic, Opt. Express 22 (2014) 16322.

[41] O. Emile, J. Emile, and H. Tabuteau, EPL 116 (2017) 64003.

[42] S. Hu, Z. W. Liao, L. Cai, and X. X. Jiang, Phys. Status Solidi 217 (2019) 1900604.

[43] M. L. Juan, M. Righini, and R. Quidant, Nat. Photonics 5 (2011) 349.

[44] Y. F. Chen, X. Serey, R. Sarkar, P. Chen, and D. Erickson, Nano Lett. 12 (2012) 1633.

[45] J. S. Huang, and Y. T. Yang, Nanomaterials 5 (2015) 1048.

[46] Z. Xu, and K. B. Crozier, Opt. Express 27 (2019) 4034.

[47] Z. Hu, J. Wang, and J. Liang, Opt. Express 12 (2004) 4123.

[48] J.-B. Decombe, S. Huant, and J. Fick, Opt. Express 21 (2013) 30521.

[49] A. Asadollahbaik, S. Thiele, K. Weber, A. Kumar, J. Drozella, H. Sterl, A. M. Herkommer, H. Giessen, and J. Fick, ACS Photonics 7 (2020) 88.

[50] X. Zhao, N. Zhao, Y. Shi, H. Xin, and B. Li, Micromachines 11 (2020) 114.

[51] M. P. MacDonald, G. C. Spalding, and K. Dholakia, Nature 426 (2003) 421.

[52] M. Woerdemann, C. Alpmann, M. Esseling, and C. Denz, Laser Photon. Rev. 7 (2013) 839.

[53] H. Rubinsztein-Dunlop, A. Forbes, M. V. Berry, M. R. Dennis, D. L. Andrews, M. J. Mansuripur, C. Denz, C. Alpmann, P. Banzer, T. Bauer, E. Karimi, L. Marrucci, M. Padgett, M Ritsch-Marte, N. M. Litchinitser, N. P Bigelow, C. Rosales-Guzmán, A. Belmonte, J. P. Torres, T. W. Neely, M. Baker, R. Gordon, A. B. Stilgoe, J. Romero, A. G. White, R. Fickler, A. E. Willner, G. Xie, B. McMorran, and A. M Weiner J. Opt. 19 (2016) 013001.

[54] O. Emile, J. Emile, and H. Tabuteau, EPL 129 (2020) 58001.

[55] E. Hecht Optics (Addison-Wesley, San Fransisco, 2001).

[56] T. M. Squires, and R. S. Quake, Rev. Mod. Phys. 77 (2005) 977.

[57] C. A. Schneider, W. S. Rasband, and K. W. Eliceiri, Nat. Methods 9 (2012) 671.

[58] O. Emile, and J. Emile, Appl. Opt. 59 (2020) 1678.

[59] A. E. Siegman Lasers (University Science Books, Sausalito CA, 1986).

[60] A. Le Floch, O. Emile, G. Ropars, and G. Agrawal, Sci. Rep. 7 (2017) 9083.

[61] A. Yildiz, J. N. Forkey, S. A. McKinney, T. Ha, Y. E. Goldman, and P. R. Selvin, Science 300 (2003) 2061.

[62] J. Vangindertael, R. Camacho, W. Sempels, H. Mizuno, P. Dedecker, and K. P. F. Janssen, Methods Appl. Fluoresc. 6 (2018) 022003.

[63] Y. Harada, and T. Asakura, Opt. Commun. 124 (1996) 529.

[64] A. Jonáš, and P. Zemanek, Electrophoresis, 29 (2008) 4813.

[65] B. K. Singh, H. Nagar, Y. Roichman, and A. Arie, Light Sci. Appl. 6 (2017) e17050.

[66] H. Nagar, T. Admon, D. Goldman, A. Eyal, and Y. Roichman, Opt. Lett. 44 (2019) 2430.

[67] www.thermofisher.com

[68] S. W. Hell, and J. Wichmann, Opt. Lett. 19 (1994) 780.

[69] S. W. Hell, Science 316 (2007) 1153. 
[70] M. R. Eftink Fluorescence quenching. In Topics in fluorescence spectroscopy J. R. Lakowicz ed. (Kluwer Academic Publishers, Dordrecht, 1992) 53.

[71] R. K. Iler, The Chemistry of Silica (Wiley, New York, 1979).

[72] G. Vigil, Z. Xu, S. Steinberg, and J. Israelachvili, J. Colloid Interface Sci. 165 (1994) 367.

[73] J. T. Verdeyen, Laser electronics, $3^{\text {rd }}$ Ed. (Prentice Hall, New York, 2001).

[74] A. Einstein, Verh. Deutsch. Phys Gesell. 18 (1916) 318.

[75] C. Cohen-Tannoudji, J. Dupont-Roc, and G. Grynberg, Atom-Photon Interactions, Basic Processes and Applications (Wiley-VCH, 1998).

[76] O. Emile, T. Galstyan, A. Le Floch, and F. Bretenaker, Phys. Rev. Lett. 75 (1995) 1511.

[77] D. Braun, and A. Libchaber, Phys. Rev. Lett. 89 (2002) 188103.

[78] A. Parola, and R. Piazza, Eur. Phys. J. E, 15 (2004) 255.

[79] S. Duhr, and D. Braun, Proc. Natl. Acad. Sci. 103 (2006) 19678.

[80] F. W. Sears, H. D. Young, and M. W. Zemansky, Uni-

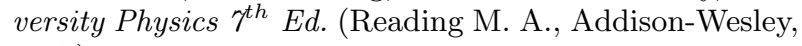
1987).

[81] M. Braibanti, D. Vigolo, and R. Piazza, Phys. Rev. Lett. 100 (2008) 108303.

[82] S. Michelin,and E. Lauga, Eur. Phys. J. E 38 (2015) 7.

[83] H. Moyses, J. Palacci, S. Sacanna, and D. G. Grier, Soft Matter 12 (2016) 6357.

[84] C. Shen, and H. D. Ou-Yang, Proc. SPIE, Optical Trapping and Optical Micromanipulation XVI 11083 (2019).

[85] M. Grzelczak, J. Vermant, E. M. Furst, and L. M. LizMarzán, ACS Nano 4 (2010) 3591.
[86] N. Vogel, M. Retsch, C. A. Fustin, A. del Campo, and U. Jonas, Chem. Rev. 115 (2015) 6265.

[87] Y. Brasse, V. Gupta, H. T. Schollbach, M. Karg, T. A. König, and A. Fery, Adv. Mater. Interfaces 7 (2020) 1901678.

[88] S. Ghosh, and A. Ghosh, Langmuir 36 (2020) 5691.

[89] M. Villangca, D. Casey, and J. Glückstad, Biophys. Rev. 7 (2015) 379.

[90] B. P. Nadappuram, P. Cadinu, A. Barik, A. J. Ainscough, M. J. Devine, M. Kang, J. Gonzalez-Garcia, J. T. Kittler, K. R. Willison, R. Vilar, P. Actis, B. Wojciak-Stothard, S.-H. Oh, A. P. Ivanov, and J. B. Edel, Nat. Nanotechnol. $14(2019) 80$.

[91] H. Zeng, P. Wasylczyk, D. S. Wiersma, and A. Priimagi, Adv. Mater. 30 (2018), 1703554.

[92] I. Shishkin, H. Markovich, Y. Roichman, and P. Ginzburg, Micromachines 11 (2020) 90.

[93] W. D. Phillips, Rev. Mod. Phys. 70 (1998) 721.

[94] O. Emile, F. Bardou, C. Salomon, P. Laurent, A. Nadir, and A. Clairon, EPL 20 (1992) 687.

[95] D. J. McCarron, E. B. Norrgard, M. H. Steinecker, and D. DeMille, New J. Phys. 17 (2015) 035014.

[96] H. Ohadi, M. Himsworth, A. Xuereb, and T. Freegarde, Opt. Express 17 (2009) 23003.

[97] E. A. Bur, R. W. Ghrist, J. C. Myatt, M. J. Holland, E. A. Cornell, and C. E. Wieman, Phys. Rev. Lett. 79 (1998) 337.

[98] M. W. Zwierlein, C. A. Stan, C. H. Schunck, S. M. F. Raupach, S. Gupta, Z. Hadzibabic, and W. Ketterle, Phys. Rev. Lett. 91 (2003) 250401. 